Review

\title{
Nanocarriers: Promising Vehicle for Bioactive Drugs
}

\author{
Manju Rawat, Deependra Singh, S. SARAF, and Swarnlata SARAF* \\ Institute of Pharmacy, Pt Ravishankar Shukla University; Raipur (C.G.), 492010 India. \\ Received May 14, 2006; accepted May 17, 2006
}

\begin{abstract}
Development of new delivery systems that deliver the potential drug specifically to the target site in order to meet the therapeutic needs of the patients at the required time and level remains the key challenge in the field of pharmaceutical biotechnology. Developments in this context to achieve desired goal has led to the evolution of the multidisciplinary field nanobiotechnology which involves the combination of two most promising technologies of 21st century-biotechnology and nanotechnology. Nanobiotechnology encompasses a wide array of different techniques to improve the delivery of biotech drugs, and nanoparticles offer the most suitable form whose properties can be tailored by chemical methods. This review highlights the different types of nanoparticulate delivery systems employed for biotech drugs in the field of molecular medicine with a short overlook at its applications and the probable associated drawbacks.
\end{abstract}

Key words nanotechnology; biotech drug; drug delivery; nanoparticle; nanobiotechnology; nanocarrier

\section{INTRODUCTION}

In recent years, the interest in sub-micron systems (i.e. nanosystems) in pharmacy has surged. This is in part due to the advantages these systems may provide over existing systems. Designing drug delivery system is challenging in terms of targeting the drug to specific sites. Certain chemicals or therapeutic agents that show success in cell culture fail to produce the same effect in the human body because of the limitation to target the designated area, as a result, high concentrations are given to patients resulting in more intense side effects. This case is similar to biopharmaceuticals and biotechnological drugs such as recombinant proteins, vaccines, antibiotics and genes, etc. However, many of them require special formulation technologies to overcome drug-associated problems such as poor solubility, drug instability in biological milieu (i.e. short half life), poor bioavailability, and potentially strong side effects that require drug enrichment at the site of action. The high-protein binding property of certain drugs hinders their passage into the brain and other organs. Suitable drug delivery systems are crucial and this is supported by a recent report stating that pharmaceuticals account for approximately $\$ 65$ billion in drug revenue every year. Poor bioavailability far too often results in not only higher patient costs and inefficient treatment, but also, more importantly, increased risks of toxicity or even death. ${ }^{1)}$

1.1. Need of Nanocarrier Nanotechnology is a novel area of science that provides, with a new hope, the tools and technology to work at atomic, molecular and supramolecular levels leading to creation of devices and delivery systems with fundamentally new properties and functions. Nanocarriers offers a number of advantages making it an ideal drug delivery vehicle (Fig. 1).

- Nanocarriers can better deliver drugs to tiny areas within the body. ${ }^{2)}$

- It represents engineering of particles, which are smaller than 100 nanometers.

- Nanotechnology is so complementary to biotechnology that promises to bridge the gaps between 'the structure' and 'the function' of biomolecules as well as between 'human physiology' and 'pathophysiology'.

- This allows the engineering of products on a comparable scale to nature such as biologicals like proteins, DNA and viruses, which are of the order of 10's of nanometers in size and cells and cellular assemblies of the order of 1000's of nanometers.

- Sophisticated techniques and tools have enabled the better characterization and manipulation of material at nanoscale level to elucidate nanoscale phenomenon leading to generation of new era of nanostructure-mediated drug delivery. Engineering on this scale enables researchers to exercise exquisite and previously unthinkable control over the physical attributes of polymers and other biomaterials.

- It is poised to help alleviate the problems of drug delivery with the development of nanostructured delivery methods in combination with utilization of principles and techniques of biotechnology to manipulate molecular, genetic, and cellular processes leading to creation of a new interdisciplinary approach.

- It involves overlap of biotech, nanotech, and information technology, might result in many important applications in life sciences including areas of gene therapy, drug delivery, imaging, biomarkers, biosensors and novel drug discovery techniques. ${ }^{3-5)}$

- It also offers an attractive solution for transformation of biosystems, and provides a broad platform in several areas of bioscience. ${ }^{6,7)}$

- Nanocarriers overcome the resistance offered by the physiological barriers in the body because efficient delivery of drug to various parts of the body is directly affected by particle size.

- Nanocarriers aid in efficient drug delivery to improve aqueous solubility of poorly soluble drugs ${ }^{8,9}$ that enhance bioavailability ${ }^{10)}$ for timed release of drug molecules, and precise drug targeting. ${ }^{11,12)}$

- The surface properties of nanocarriers can be modified for targeted drug delivery ${ }^{12-14)}$ for e.g. small molecules, proteins, peptides, and nucleic acids loaded nanoparticles are not recognized by immune system and efficiently targeted to particular tissue types. ${ }^{15)}$

- Targeted nano drug carriers reduce drug toxicity and provide more efficient drug distribution. ${ }^{16)}$

- Nanocarriers holds promise to deliver biotech drugs over various anatomic extremities of body such as blood brain 


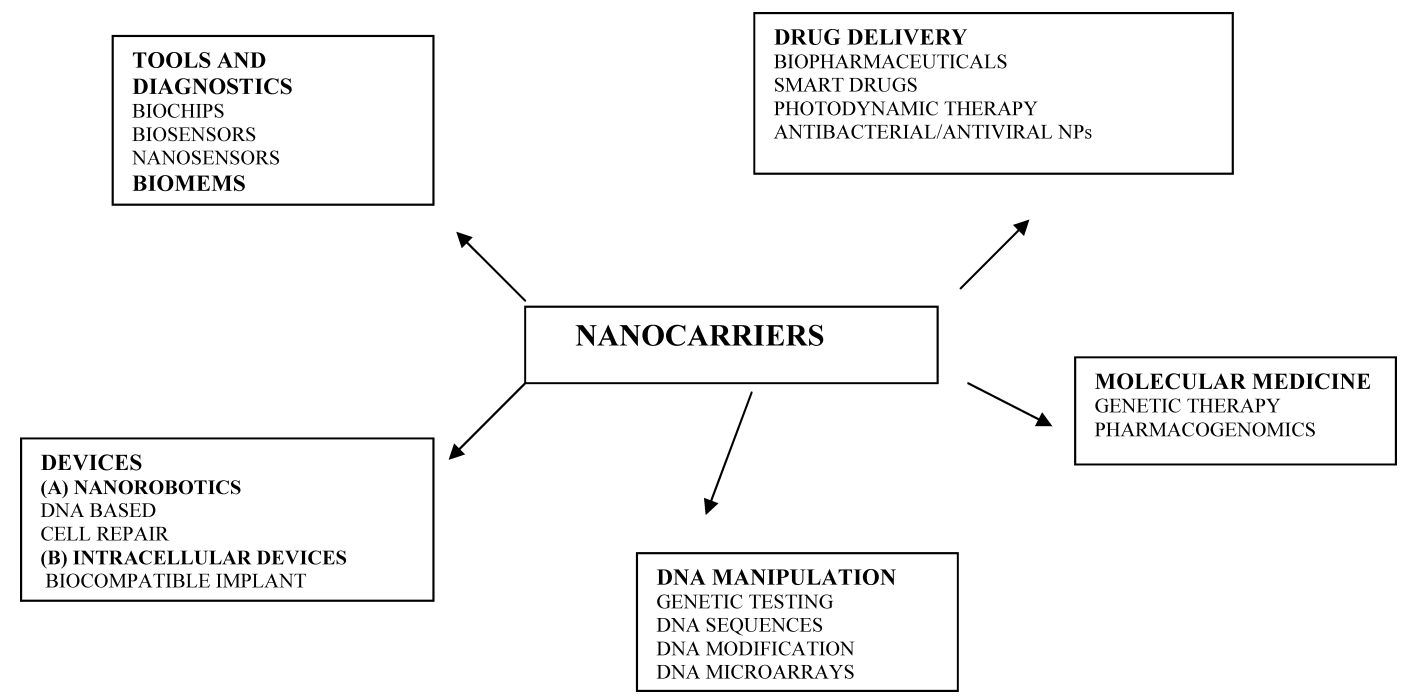

Fig. 1. Multidisciplinary Functions of Nanocarriers

barrier, branching pathways of the pulmonary system, and the tight epithelial junctions of the skin etc.

- Nanocarriers better penetrate tumors due to their leaky constitution, containing pores ranging from $100-1000 \mathrm{~nm}$ in diameter.

\subsection{Limitations}

- Nanocarriers exhibits difficulty in handling, storage, and administration because of susceptibility to aggregation.

- It has unsuitability for less potent drugs.

- But the key area of concern is related to its small size as nanocarriers can gain access to unintended environments with harmful consequences, e.g. it can cross the nuclear envelope of a cell and cause unintended genetic damage and mutations. ${ }^{17)}$

\section{TYPES OF NANOCARRIERS}

Nanocarriers are materials or devices of nanoscale (below $1 \mu \mathrm{m})$ made up of different biodegradable materials like natural or synthetic polymers, lipids or phospholipids and even organometallic compounds. Nanocarriers being of submicron size have a very high surface to volume ratio, leading to increased dissolution rate. RES is usually responsible for the uptake of nanoparticles in the body. ${ }^{13)}$ But, this uptake is related to properties of the nanocarriers such as size and the surface properties. Nanocarriers include a wide array of submicron system such as nanoparticles, nanocapsules, lipid complexes, polymeric micelles, and dendrimers ${ }^{4,16)}$ (Table 1 , Fig. 2).

2.1. Nanocrystals and Nanosuspensions Nanocrystals are aggregates of around hundreds or thousands of molecules that combine in a crystalline form, composed of pure drug with only a thin coating comprised of surfactant or combination of surfactants. The production technique of nanocrystals is known as 'nanonisation'. 18) To produce nanosuspensions, the drug powder is dispersed in an aqueous surfactant solution by high speed stirring. The obtained macrosuspension is then homogenized to nanosize by wet milling, ${ }^{19)}$ high-pressure homogenization, ${ }^{20)}$ nanocrystallisation from supersaturated solution ${ }^{21)}$ and spray drying. ${ }^{22)}$ Characterization of obtained nanoparticles is done in terms of high weight per volume, which renders them insoluble in either lipophilic or hydrophilic media.

Problems typical of poorly soluble drugs like reduced bioavailability, improper absorption pattern and problems of preparing the parenteral dosage form may be resolved by formulation as nanocrystals. This has several benefits, unlike carrier-based nanoparticles in which extent of loading may be low. Only a minimum quantity of surfactants needs to be added in nanocrystals for steric and electrostatic surface stabilization. Moreover, administration of high drug levels with depot release can be achieved if dissolution is sufficiently slow. This further helps to achieve a high $A U C$ with reduced $C_{\max }$ and toxicity. As pure drug is used and no carrier is needed, eliminating potential toxicity issues associated with the carrier molecule. Nanocrystal technology can be utilized for many dosage forms. Nanoparticles offer the potential for targeting the mucosa of the gastrointestinal tract after oral administration, and targeting the cells of the mononuclear phagocytic system (MPS) to treat infections of the MPS such as fungal mycobacterial infections and leishmaniasis, thus serving as a favourable delivery system for drugs like amphotericin B, tacrolimus, etc. ${ }^{20)}$ The size of nanocrystals allows for safe and effective passage through capillaries. Potential of nanocrystals can be inferred by the FDA approval of Rapamune ${ }^{\mathbb{R}}$, containing sirolimus which is an immunosuppressant drug to prevent graft rejection in children after liver transplantation and Emend ${ }^{\circledR}$, which contains aprepitant, MK 869, is used in the treatment of emesis associated with the cancer chemotherapy.

2.2. Nanotubes and Nanowires Nanotubes and nanowires are the self-assembling sheet of atoms arranged in the form of tubes and thread-like structures of nanoscale range. ${ }^{23)}$ Nanostructures that have gained much attention are hollow, carbon-based cage like structures - nanotubes and fullerenes. Fullerenes are spherical structures, also known as bucky balls. Soluble derivatives of fullerenes such as $\mathrm{C}_{60}-\mathrm{a}$ soccer ball shaped arrangement of 60 carbon atoms per molecule shows promise as pharmaceutical agents. These synthetic nanocarriers offer a number of advantages in terms of increased internal volume and ease of functional modification of internal and external surfaces. ${ }^{24)}$ Nanotubes are of two 


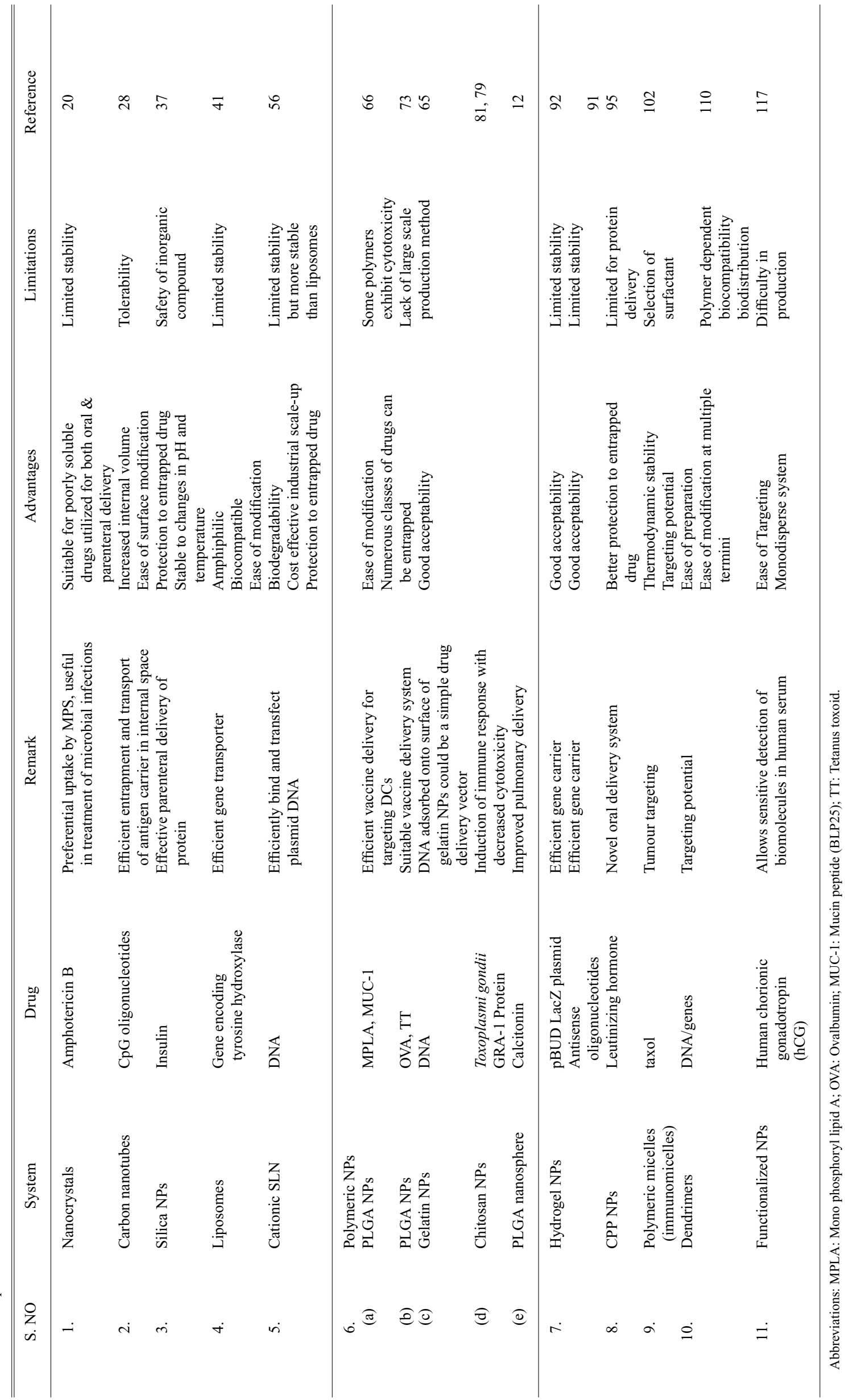




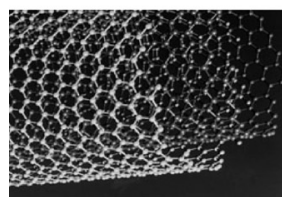

(a) nanotubes

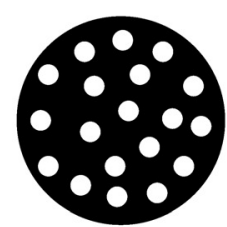

d (i) nanospheres

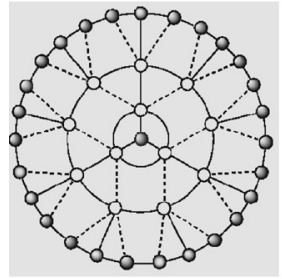

(f) dendrimers

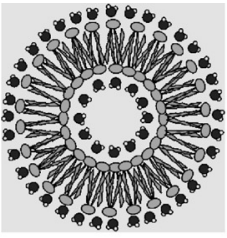

(b) liposomes

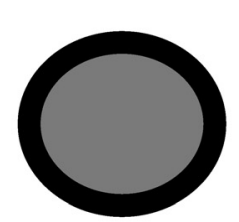

d (ii) nanocapsules

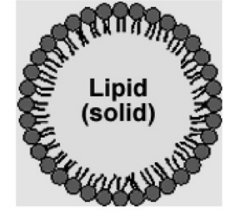

(c) SLN

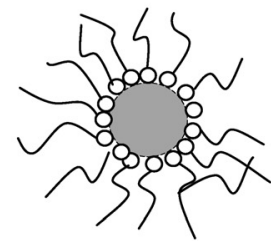

(e) Polymeric micelles
Catalytic

active sites

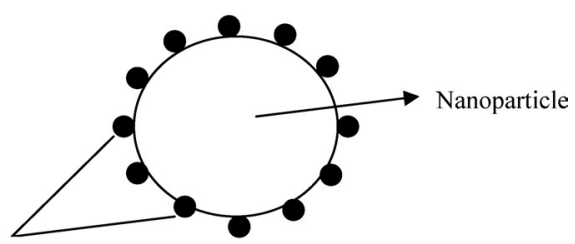

(g) Functionalized nanoparticles

Fig. 2. Different Types of Nanocarriers

(a) Nanotubes: self-assembling lipid tubes. (b) Liposomes: concentric bilayered vesicles in which an aqueous volume is entirely enclosed by a membranous lipid bilayer. (c) Solid lipid nanoparticles (SLN): submicron colloidal carriers made from solid lipids. (d) Polymeric nanoparticles. (i) Nanospheres: nanoparticles in which drug is dispersed through out the polymeric matrix. (ii) Nanocapsules: nanoparticles in which drug is encapsulated within polymeric membrane. (e) Polymeric micelles: amphiphilic block copolymers that self-associate in aqueous solution. (f) Dendrimers: macromolecular compounds that consist of a series of branches around an inner core. (g) Functionalized nanoparticles: monodisperse-sized particles of uniform shape with well-defined surface composition.

types-single walled and double walled carbon tubes. Fullerenes and carbon nanotubes are prepared by various techniques such as electric arc discharge, laser ablation, chemical vapour deposition or combustion processes. ${ }^{25}$ )

Among the category of nanotubes single walled carbon nanotubes offer the most promising approach for gene and drug delivery system as their physical dimensions mimic nucleic acids. Recent work favoring their role as gene transporters involved coating nanotubes with ribonucleic acid polymer or bovine serum albumin to reduce its hydrophobicity. Carbon nanotubes are characterized in terms of diffusion by single molecule fluorescent microscopy. ${ }^{26)}$ These synthetic carriers were found to be biocompatible when studied on human HEK 293 cells, signifying their safety. ${ }^{27)}$ Multiwalled carbon nanotubes are also suitable delivery system for transformation specifically to bacterial cells (E. coli) and for nanoscale cell electroporation. ${ }^{28)}$ Pantarotto et al. demonstrated the potential of peptide functionalized carbon nanotubes in augmentation of virus specific neutralizing antibody response that could be further exploited in vaccine delivery. ${ }^{29)}$ In another work, hybrid of gelatin hydrogel with carbon nanotubes imparted stability to the hydrogel at $37^{\circ} \mathrm{C}$, and thus safe for delivery of proteins and peptides. ${ }^{30}$ Fullerenes are effective in tissue selective and intracellular targeting of mitochondria. ${ }^{31)}$ Thus, these systems could be utilized further for targeting capabilities of biotech drugs such as genes, proteins and peptides.

2.3. Ceramic Nanoparticles These are the nanoparti- cles made up of inorganic (ceramic) compounds such as silica, titania and alumina. Ceramic nanoparticles exist in size less than $50 \mathrm{~nm}$, which helps them in evading reticuloendothelial system (RES) of body. These particles provide the complete protection to the entrapped molecules such as proteins, enzymes and drugs against the denaturizing effects of external $\mathrm{pH}$ and temperature as it involves no swelling and porosity changes with the change in $\mathrm{pH}^{34)}$ Moreover, these exhibit properties of compatibility ${ }^{32}$ and ease of surface modification $^{33)}$ for effective targeting. Luo and co-workers ${ }^{34)}$ demonstrated utility of silica nanoparticles with covalently linked cationic surface modifications in efficient binding, condensation and protection of plasmid DNA with threecomponent transfection system that might be utilized efficiently in DNA transfection. These are effective in the delivery of proteins and genes. ${ }^{32,35,36)}$ In yet another work by Paul and Sharma porous hydroxyapatite nanoparticles entrapped in alginate matrix containing insulin for oral administration demonstrated the desirable controlled release of the protein signifying further use of ceramic nanoparticles in delivery of proteins and peptides. ${ }^{37)}$ But these ceramic nanoparticles are non-biodegradable, slow dissolving and nonetheless must be somehow eliminated from the body.

2.4. Liposomes Liposomes are concentric bilayered vesicles in which an aqueous volume is entirely enclosed by a membranous lipid bilayer mainly composed of natural or synthetic phospholipids. Liposomes are formed when thin lipid films or lipid cakes are hydrated and stacks of liquid 
crystalline bilayers become fluid and swell. During agitation hydrated lipid sheets detach and self associate to form vesicles, which prevent interaction of water with the hydrocarbon core of the bilayer at the edges. Small unilamellar vesicles (SUVs) are surrounded by single lipid layer $(25-50 \mathrm{~nm})$ whereas several lipid layers separated by intermittent aqueous layer surround large unilamellar vesicles (LUV). Liposomes are characterized in terms of size, surface charge and number of bilayers. It exhibits number of advantages in terms of amphiphilic character, biocompatibility, and ease of surface modification rendering it a suitable candidate delivery system for biotech drugs. Liposomes have been used successfully in the field of biology, biochemistry and medicine since its origin. ${ }^{38)}$ These alter the pharmacokinetic profile of loaded drug to a great extent especially in case of proteins and peptides ${ }^{39)}$ and can be easily modified by surface attachment of polyethylene glycol-units (PEG) making it as stealth liposomes and thus increase its circulation half-life. ${ }^{40)}$ Similar approach was utilized by packaging therapeutic molecules inside a liposome and decorating the surface of liposome using molecular "Trojan Horse" technology, Zhang et al., prepared OX-26-transferrin-targeted polyethylene glycol (PEG)ylated immunoliposomes carrying expression plasmids of gene encoding tyrosine hydroxylase and promising results were obtained in a rat model of Parkinson's disease. ${ }^{41)}$ This could also be exploited in target specific delivery of potent drugs and genes.

2.5. Solid Lipid Nanoparticles (SLN) Another lipidbased formulation comprises solid lipid nanoparticles that employ the use of solid lipids. SLN particles made from solid lipids are submicron colloidal carriers $(50-1000 \mathrm{~nm})$ dispersed either in water or in an aqueous surfactant solution. These consist of solid hydrophobic core having a monolayer of phospholipid coating. The solid core contains drug dissolved or dispersed in the solid high melting fat matrix. The hydrophobic chains of phospholipids are embedded in the fat matrix. Depending on the type and concentration of the lipid, 0.5 to $5 \%$ emulsifier (surfactant) is added for the physical stabilization of the system. In particular poloxamer 188 , polysorbate 80 , lecithin, polyglycerol methylglucose distearate, sodium cocoamphoacetate or saccharose fatty acid esters are very often employed. SLN are prepared by various techniques ${ }^{42)}$ such as high-pressure homogenization, ${ }^{43}$ ) microemulsion formation, ${ }^{44)}$ precipitation, ${ }^{45)}$ and as lipid nanopellets ${ }^{46)}$ and lipospheres. ${ }^{47,48)}$ Speiser reported initial work on SLN in 1990 on the oral delivery of lipid nanopellets. Being in the solid state, the lipid components of SLN degrade more slowly and thus provide long lasting exposure to the immune system. ${ }^{46}$ Olbrich et al. studied the effect of surfactant and lipid composition on the degradation time of SLN and found that the use of sterically stabilizing surfactants can promote delay in degradation by hindering the anchorage of enzyme complexes. ${ }^{49,50)}$

Different delivery routes have been exploited such as parenteral, ${ }^{51)}$ pulmonary $^{52)}$ and topical. ${ }^{53,54)}$ SLN are non-toxic when compared with polymeric nanoparticles. ${ }^{55)}$ Cationic solid lipid nanoparticles can serve as an effective, potent non-viral transfection agent. ${ }^{56,57)}$ SLN offers a futuristic approach as an effective adjuvant for vaccines to give a maximum immune response by optimizing surface properties. In a recent work, chitosan coated lipid nanoparticles for oral salmon calcitonin delivery were prepared which can be used for oral administration of peptidal drugs. ${ }^{58)}$ This also serves as an effective alternative to existing systems in terms of biodegradability, good tolerability and availability of cost-effective industrial scale-up techniques such as high-pressure homogenization, and microemulsion technology, thereby paving its way to pharmaceutical industry.

2.6. Polymeric Nanoparticles Colloidal carriers based on biodegradable and biocompatible polymeric systems have largely influenced the controlled and targeted drug delivery concept. ${ }^{59)}$ Nanoparticles are sub-nanosized colloidal structures composed of synthetic or semi-synthetic polymers that vary in size from $10-1000 \mathrm{~nm}$. Depending upon the method of preparation, nanospheres, or nanocapsules can be obtained in which drug either is dissolved, entrapped, encapsulated or attached to the nanoparticle matrix. Polymeric materials exhibit several desirable properties including biocompatibility, biodegradability, surface modification, and ease of functionalization of polymers. Polymeric systems allow for a greater control of pharmacokinetic behaviour of the loaded drug, leading to more appropriate steady levels of drugs. ${ }^{60)}$ These attributes make it a candidate system for effective entrapment or encapsulation of biotech drugs that are usually sensitive to the changes in the surroundings. ${ }^{61)}$

Biodegradable polymeric nanoparticles, typically consisting of polylactic acid (PLA), polyglycolic acid (PGA), polylactic-glycolic acid (PLGA), and polymethyl methacrylate (PMMA) are being investigated for the delivery of proteins, genes and DNA. ${ }^{62-65)}$ PLGA Poly-(D,L-lactic-co-glycolic acid) based nanoparticles have also proven their ability in enhanced delivery of antigens to dendritic cells. ${ }^{66)}$ Biodegradable polymeric nanoparticles offer an effective oral vaccine delivery system with an increased $\operatorname{IgA}$ antibody response and thus hold promise for oral vaccination. ${ }^{67-69)}$ Nanoparticles have also been utilized in the delivery of antisense oligonucleotides, susceptible to degradation by nucleases. ${ }^{70-72)}$ Polyalkyl cyanoacrylate nanoparticles and nanocapsules were developed as peptide carriers for insulin, ${ }^{73)}$ PGDF-Receptor $\beta$ tyrphostin inhibitor, ${ }^{74)}$ Interferon- $\alpha,{ }^{75)}$ and tumor necrosis factor- $\alpha{ }^{76)}$ Polymeric nanoparticle bound peptides can be used for sustained oral delivery and also to improve absorption and bioavailability. ${ }^{77)}$

Water based polymeric biodegradable delivery systems have been proposed for efficient delivery of peptides. ${ }^{78)}$ Natural polymers like chitosan, gelatin, albumin, and sodium alginate have also been tried to avoid the toxicological problems associated with the use of synthetic polymers. ${ }^{70,79-81)}$ Recently FDA approved drug made up of albumin nanoparticles is Abraxane ${ }^{\circledR}$ containing paclitaxel for use in patients with metastatic breast cancer who have failed combination therapy. In yet another work relating to chitosan nanoparticles (NPs), Toxoplasma gondii GRA1 protein and DNA vaccine were loaded onto chitosan NPs and results demonstrated its role in efficient oral delivery of vaccines. ${ }^{79)}$ Protein, peptides, vaccines, and oligonucleotides represent an important class of biotech drugs for which polymeric nanoparticles offer an attractive possibility. ${ }^{60,82,83)}$

Polymeric nanoparticles offer engineered specificity allowing them to deliver a higher concentration of pharmaceutical agent to a desired location. But, particulate drug carriers are subject to rapid removal from the circulation by the 
macrophages of MPS, the main obstacle in targeting various non-phagocytic cells of the body. Attempts have been made to develop stealth nanoparticles carrying proteins and peptides by surface modification with a hydrophilic, flexible and non-ionic polymer, like poly(ethylene glycol), which has shown promising results. ${ }^{76,84)}$ In spite of numerous advantages offered by the polymeric nanoparticles due to the availability of an extensive number of polymers, they also have limitations in terms of its cytotoxicity, because their nanometric size range is conducive to internalization by cells (macrophages), and degradation inside the cell can lead to cytotoxic effects as reported for polyester polymers. ${ }^{85}$ ) Another limitation is the lack of suitable large-scale production methods. Thus a lot of effort is still needed to synthesize newer polymers and copolymers to match the hydrophilic and hydrophobic properties of the drug together with suitable large-scale production methods for effective formulation.

2.7. Hydrogel Nanoparticles Hydrogel nanoparticles is another polymeric system involving the self-assembly and self aggregation of natural polymer amphiphiles such as hydrophobized polysaccharides like cholesteroyl pullulan, cholesteroyl dextran and agarose where cholesterol groups provide cross linking points in a non-covalent manner. Changing the degree of substitution of cholesterol groups can modulate the size and density of hydrogel nanoparticles. ${ }^{86-88)}$

Stimuli responsive hydrogels have been prepared, which respond to changes in $\mathrm{pH}$ and temperature, and have been found to be tissue compatible ${ }^{88,89)}$ Recently, Pattou and Palmer demonstrated the utility of a novel type of temperature responsive oxygen carrier that was prepared using poly( $N$-isopropyl acrylamide) hydrogel nanoparticles encapsulating bovine hemoglobin which might benefit tissue hypoxia caused by decreased body temperature. ${ }^{90)}$ Hydrogel nanoparticles have been utilized in the effective delivery of antigens, DNA and antisense oligonucleotides. ${ }^{91-93)}$ Cross-linked hydrogel nanoparticles (PVP-NP) $(35-50 \mathrm{~nm}$ in diameter) composed of natural polymers offers targeting to intracellular sites and good acceptability because of higher water content.

2.8. Copolymerized Peptide Nanoparticles (CPP) Another modification of a polymer-based system is copolymerized peptide nanoparticles. It is a novel approach utilized for delivery of therapeutic peptides as drug-polymer conjugates in which the drug moiety is covalently bound to the carrier instead of being physically entrapped. In 1996, Hillery et $a l .{ }^{94)}$ reported in vitro stability of CPP of leutinizing hormone releasing hormone (LHRH), and results demonstrated the significant intestinal transport of associated hormone (LH) using Caco-2 cell lines. Ramnathan et al. ${ }^{95)}$ further supported the efficiency of this conjugated system. Review in this context is also available. ${ }^{96)}$ This system needs to be further explored for effective delivery of sensitive molecules such as peptides and proteins.

2.9. Polymeric Micelles These systems include amphiphilic block copolymers such as Pluronics (polyoxyethylene polyoxypropylene block copolymers that self-associate in aqueous solution to form micelles. These are characterized by size and surface properties. Polymeric micelles offer a number of advantages in terms of thermodynamic stability in physiological solution leading to their slow dissolution in vivo. ${ }^{97,98)}$ Because of their core-shell structure, these serve as suitable carrier for water insoluble drugs, such drugs parti- tion in the hydrophobic core of micelles and outer hydrophilic layer aids in dispersion in aqueous media making it an appropriate candidate for intravenous administration. ${ }^{99}$ Nanometric size range helps micelles to evade the RES, and aids passage through endothelial cells. ${ }^{100}$ )

Polymer micelles have been extensively studied as drug carriers. ${ }^{101)}$ Conjugating to ligands such as antibodies can enhance targeting potential of micelles. Immunomicelles is one such novel approach in which antibody conjugated polymeric micelles containing antitumor drug Taxol was prepared and results demonstrated effective delivery at tumour site. ${ }^{102)}$ Targeting has also been achieved in other drugs with reduced toxicity. ${ }^{103)}$ Novel polymeric micelles with targetability and stimuli sensitivity have emerged as promising carriers in gene and drug delivery, and can potentially establish landmarks in the future of drug delivery systems. ${ }^{104)}$

2.10. Dendrimers Dendrimers are the macromolecular compounds that consist of a series of branches around an inner core whose size and shape can be altered as desired. These represent a unique class of polymers that are fabricated from monomers using either convergent or divergent step growth polymerization. ${ }^{105)}$ Dendrimers are made from $\mathrm{AB}_{n}$ type monomers, each layer or generation of branching units doubles or triples $(n=2, n=3)$ the number of peripheral functional groups. Generally during dendrimer formation molecules emanate from a core and like a tree they ramify with each subsequent branching unit referred to as generation. Drug molecules can be loaded either in the interior, or can be adsorbed or attached to the surface groups. Dendrimers are made up of different types of polymers such as polyamidoamine (PAMAM), poly(L-glutamic acid), polyethyleneimine, polypropyleneimine, and polyethylene glycol. ${ }^{106)}$

It offers enormous advantages such as nanometric size range, ease of modification by modifying their termini, ease of preparation, and availability of multiple copies of surface groups for biological reorganization processes. ${ }^{107,108)}$

Hydrophilic dendrimers are suitable as coating agents for protection and delivery of drugs to specific sites, thus minimizing drug toxicity. ${ }^{109)}$ In a recent work by Choi et al. ${ }^{110)}$ DNA assembled polyamidoamine dendrimer clusters were prepared for cancer cell specific targeting. Polyamidoamine dendrimers have demonstrated successful gene transfection and transepithelial transport efficiency. ${ }^{111,112)}$ However, application of dendrimers as a potential drug carrier requires focus on improvement in the design of the dendritic framework in terms of biocompatibility and biodistribution.

2.11. Functionalized Nanocarriers The combination of functionalities of biomolecules and non-biologically derived molecular species used for special functions such as markers for research in cell, molecular biology, biosensing, bioimaging and marking of immunogenic moieties to targeted drug delivery are known as functionalized nanoparticles. ${ }^{113)}$ These carriers are monodisperse-sized particles of uniform shape with well-defined surface composition. Nanoparticles from catalytically active metals such as platinum, palladium and silver and non-catalytically active metals such as gold have been prepared for functionalization purposes. Organically functionalized nanoparticles of catalytic active metals offer a high surface area and unique size dependent chemical behaviour. One approach is the bioconjugate quantum dots as fluorescent biological labels. Quan- 
tum dots are crystalline clumps of several hundred atoms with an insulating outer shell of a different material. ${ }^{114}$ ) Quantum dots can be attached to the biologicals such as cells, proteins and nucleic acids. These can be constructed to emit light at different wavelengths extending from ultraviolet to infrared range. Light emitted by these quantum dots is so bright that it is possible to detect even the cellular or subcellular structures. Quantum dots, being of inorganic origin, make the system stable, and their inert coating at inner surfaces makes them less toxic than dyes of organic origin. Thus quantum dots can serve as an effective delivery and diagnostic agent to detect quantitatively the cellular contents. Another such approach is PEBBLES (Probes Encapsulated by Biologically Localized Embedding), which has been utilized in monitoring cell metabolism and disease conditions. ${ }^{115)}$ DNA-based gold nanoparticles have demonstrated the ability to detect specific DNA sequences and this strategy is further extended to analyze combinatorial DNA arrays (genechips). ${ }^{116)}$ Furthermore, functionalized nanoparticles have been used as optical sensors to quantitatively assay biomolecules such as human chorionic gonadotropin (hCG) in human serum. ${ }^{117)}$ Darkow et al. demonstrated removal of endotoxins from water, dialysis fluid, plasma or blood by using functionalized nanoparticles. ${ }^{118)}$ Another approach is the solid nanoshell polymer composite, which is a photo-thermally triggered drug delivery system. ${ }^{119,120)}$ Bio MEMS which refers to Biomedical or Biological Micro Electro Mechanical systems, covers a profound field of work such as diagnosis, therapeutics and tissue engineering. ${ }^{121)}$ Biomolecular conjugation methods of metals include bifunctional linkages, lipophilic interaction, silanization, electrostatic attraction and nanobead interaction. Hybrid biomolecular components are promising in the field of nanobiotechnology. ${ }^{122)}$

\section{CONCLUSION}

Utilization of nanotechnological approaches for the delivery of biotech drugs is rapidly becoming an important tool in the arsenal of drug delivery. This multidisciplinary approach offers a great deal of flexibility in terms of ease of modification and adaptation to meet the needs of pathological conditions. Based on the review of the available literature, it could be inferred that although numerous drug delivery approaches are available none of them seems perfect and suffers from one limitation or another (Table 1). More studies on the toxicological effects of nanoparticles in humans need to be done. Extensive research is needed to overcome the limitations associated with these systems as it could allow the direct treatment of the cause of disease rather than its symptoms, because of the accessibility to otherwise inaccessible intracellular targets. Nanobiotechnology brings a new ray of hope for the delivery of biotech drugs that exhibit the problems of short half-life, poor bioavailability, strong side effects, insolubility and instability in biological milieu. Nanobiotechnology, although still in its infancy, has proven its ability to overcome the barriers involved in the delivery of biotech drugs and its success and acceptability can be implicated through FDA approval of Abraxane ${ }^{\circledR}$ (albumin bound paclitaxel), Rapamune ${ }^{\circledR}$ (sirolimus), and Emend ${ }^{\circledR}$ (aprepitant, MK 869). Moreover with the advent of novel nanoparticulate systems and their success in delivery of biotech drugs, it is poised to play a profound and dominant role in the field of molecular medicine and associated delivery of drugs in the future.

Acknowledgement The authors are thankful to all the colleagues of Biotech Lab. Institute of Pharmacy, Pt Ravishankar Shukla University, Raipur (C.G.) India for their valuable suggestions and help.

\section{REFERENCES AND NOTES}

1) Glen A.: 〈http://www.nanomarkets.net $\rangle$, The impact of nanotecnology in drug delivery: Global developments, market analysis and future propects, 2005.

2) Roco M. C., Williams R. S., Alivisatos P. (eds.), Biological, medical and health applications. "Nanotechnology Research Directions," Chap. 8, Kluwer Academic Publishers, Boston, 2000. URL: http://nano.gov.

3) Roco M. C., Curr. Opin. Biotechnol., 14, 337-343 (2003).

4) Sahoo K. S., Labhasetwar V., Drug Discov. Today, 8, 1112-1120 (2003).

5) Wilkinson J. M., Med. Device Technol., 14, 29-31 (2003).

6) Bugunia-Kubik K., Susisaga M., J. Biosystem, 65, 123-138 (2002).

7) Schimidt J., Montemagno C., Drug Discov. Today, 7, 500-503 (2002).

8) Kipp J. E., Int. J. Pharm., 284, 109-122 (2004).

9) Ould-ouali L., Noppe M., Langlois X., Willems B., Riele P. T., Timmerman P., Brewster M. E., Arien A., Preat V., J. Control. Rel., 102 657-668 (2005).

10) Arbos P., Campaner O., Miguel A., Arangoa Miguel A., Irache J. M., J. Contr. Rel., 96, 55-65 (2004).

11) Dubin C. H., Mech. Enz. Nanotechnol., 126 (Suppl.), 10-12 (2004).

12) Yamamoto H., Kuno Y., Sugimoto S., Takeuchi H., Kawashima Y., J. Contr. Rel., 102, 373-381 (2005)

13) Peppas L. B., Blanchette J. O., Adv. Drug Del. Rev., 56, 1649-1659 (2004).

14) Kreuter J., Adv. Drug Del. Rev., 47, 65-81 (2001).

15) Cohen H., Levy R. J., Gao J., Fishbein I., Kousaev V., Sosnowski S., Slomkowski S., Golomb G., Gene Ther., 7, 1896-1905 (2000).

16) Lobenberg R., Kreuter J., AIDS Res. Hum. Retroviruses, 12, 1709 1715 (1996)

17) Hoet Peter H. M., Hohlfeld I. B., Salata O. V., J. Nanobiotechnol., 2 , $1-5$ (2004).

18) Rabinow Barrett E., Nat. Rev. Drug Discov., 3, 785-796 (2004).

19) Liversidge E. M., Liversidge G. G., Cooper E. R., Eur. J. Pharm. Sci., 18, 113-120 (2003).

20) Muller R. H., Jacobs C., Kayser O., Adv. Drug Del. Rev., 47, 3-19 (2001).

21) Sarkari M., Brown J., Chen X., Swinnea S., Williams Robert O., Johnston Keith P., Int. J. Pharm., 243, 17-31 (2002).

22) Chow L. C., Sun S., Hockey B., J. Res. Natl. Inst. Stand. Technol., 109, 543-551 (2004)

23) Martin C. R., Kohli P., Nat. Rev. Drug Discov., 2, 29-37 (2003).

24) Goldstein A. S. M., Amory J. K., Martin S. M., Vemon C., Matrumotom A., Yagerm P., Bioorg. Med. Chem., 9, 2819-2825 (2001).

25) Mamalis A. G., Vogtlander L. O. G., Markopoulos A., Precision Eng., 28, 16-30 (2004).

26) Lu Q., Freedman K. O., Rao R., Huang G., Lee J., Larcom L. J., Raom A. M., Ke P. C., J. Appl. Phys., 96, 6772-6775 (2004).

27) Daxiang C., Furong T., Cengiz S. O., Mao W., Huajian G., Toxicology Lett., 155, 73-85 (2005).

28) Rojas C. J., Troszczynska J., Firkowska I., Morsczeck C., Giersig M., Lab. Chip., 5, 536-539 (2005).

29) Pantarotto D., Partidos C. D., Hoebeke J., Brown F., Kramer E., Briand J., Muller S., Prato M., Bianco A., Chem. Biol., 10, 961-966 (2003).

30) Li H., Wang D. Q., Liu B. L., Gao L. Z., Colloids and Surfaces B: Biointerfaces, 33, 85-88 (2004).

31) Foley S., Crowley C., Smaihi M., Bonfils C., Erlanger B. F., Seta P., Larroque C., Biochem. Biophys. Res. Commun., 294, 116-119 (2002). 
32) Jain T. K., Roy I., De T. K., Maitra A., J. Am. Chem. Soc., 120, $11092-11095$ (1998).

33) Badley R. D., Warren T. F., Mcenroe F. J., Assink R. A., Langmuir, 6, $792-801$ (1990).

34) Luo D., Han Et., Belcheva N., Saltzman W. M., J. Control. Rel., 95, 333-341 (2004)

35) Cherian A. K., Rana A. C., Jain S. K., Drug Del. Ind. Pharm., 26, 459-463 (2000)

36) Roy I., Mitra S., Maitra A., Mozumdar S., Int. J. Pharm, 250, 25-33 (2003).

37) Paul W., Sharma C. P., Trend Biomat. Artific Org., 14, 37-38 (2001).

38) Bangham A. D., Standish M. M., Watkins J. C., J. Mol. Biol., 13, $238-252$ (1965).

39) Allen T. M., Drugs, 54 (Suppl. 4), 8-14 (1997).

40) Lasic D. D., Vallner J. J., Working P. K., Curr. Opin. Mol. Ther, 1 177-185 (1999).

41) Zhang Y., Calon F., Zhu C., Boado R. J., Pardridge W. M., Hum. Gene Ther., 14, 1-12 (2003).

42) Wolfgang M., Karsten M., Adv. Drug Del. Rev., 47, 165-196 (2001).

43) Muller R. N., Schwarz C., Metinert W., Lucks J. S., Proc. Int. Symp. Control. Release Bioact. Mater., 20, 480-481 (1993).

44) Gasco M. R., US. Patent 5250236 (1993).

45) Siekmann B., Westesen K., Eur. J. Pharm. Biopharm., 43, 104-109 (1996).

46) Speiser P., European Patent EP 0167825 (1990)

47) Domb A. J., Int. J. Pharm., 124, 271-278 (1995).

48) Cortesi R., Esposito E., Luca G., Nastruzzi C., Biomaterials, 23 , 2283-2294 (2002)

49) Olbrich C., Mehnert W., Muller R. H., J. Proc. Int. Symp. Control. Release Bioact. Mater, 24, 921-922 (1997).

50) Olbrich C., Muller R. H., Int. J. Pharm., 180, 31-39 (1999).

51) Wissing S. A., Kayser O., Muller R. H., Adv. Drug Del. Rev., 56, $1257-1272$ (2004).

52) Sanna V., Kirschvink N., Gustin P., Gavini E., Roland I., Delattre L., Evrard B., AAPS Pharm. Sci. Tech., 5, 1-7 (2003).

53) Muller R. H., Dinglet A., Eurocosmetics, 7, 19-26 (1998)

54) Jenning V., Gysler A., Korting M. S., Gohla S. H., Eur. J. Pharm. Biopharm., 49, 211-218 (2000).

55) Muller R. H., Maaben S., Weyhers H., Specht F., Lucks J. S., Int. J. Pharm., 138, 85-94 (1996).

56) Olbrich C., Bakowsky U., Lehr C. M., Muller R. H., Kneuer C., J. Control. Rel., 77, 345-355 (2001).

57) Pedersen N., Hansen S., Heydenreich A. V., Kristensen H. G., Poulsen H. S., Eur. J. Pharm. Biopharm., 62, 155-162 (2006)

58) Garcia-Fuentes M., Torres D., Alonso M. J., Int. J. Pharm., 296, $122-132$ (2005)

59) Vyas S. P., Khar R. K., "Targeted and Control Drug Delivery,” 1st ed., Chap. 9, CBS Publishers and Distributors, New Delhi, 2002, pp. $331-385$.

60) Peppas L. B., Int. J. Pharm., 116, 1-9 (1995).

61) Soppimath K. S., Aminabhavi T. M., Kulkarni A. R., Anandrao R. Rudzinski W., Eur. J. Control. Rel., 70, 1-20 (2001).

62) Borchard G., Kreuter J., J. Drug Target., 1, 15-19 (1993).

63) Stieneker F., Kreuter J., Lower J., Vaccine Res., 2, 111-118 (1993).

64) Panyam J., Labhasetwar V., Adv. Drug Deliv. Rev., 55, 329-347 (2003).

65) Zillies J., Coester C., J. Pharm. Pharm. Sci., 7, 17-21 (2004).

66) Elamanchili P., Diwan M., Cao M., Samuel J., Vaccine, 22, 2406 2412 (2004)

67) Delie F., Blanco-prieto M. J., Molecules, 10, 65-80 (2005)

68) Mutwiri G., Bowersock T. L., Babiuk L. A., Expert Opin. Drug Deliv., 2, 791-806 (2005).

69) Sergio A., Rodriguez G., Critical Reviews ${ }^{\mathrm{TM}}$ Therapeutic Drug Carrier Systems, 22, 419-464 (2005).

70) Aynie I., Vauthier C., Chacun H., Fattal E., Couvreur P., Antisen Nucleic Acid Drug Dev., 9, 301-302 (1999).

71) Hughes M. D., Hussain M., Nawaz Q., Sayyed P., Jain T. K., Roy I., De T. K., Maitra A., J. Am. Chem. Soc., 120, 11092-11095 (1998).

72) Lochmann W. D., Zimmer A., J. Control. Rel., 100, 411-423 (2004).

73) Prokop A., Kozlov E., Newman G. W., Newman M. J., Biotech. Bioeng., 78, 459-466 (2002).

74) Michel C., Aprohamiam M., Defontaine L., Couvreur P., Damage C., J. Pharm. Pharmacol., 43, 1-5 (1991).

75) Fishbein I., Chorny M., Rabinovich L., Banai S., Gati I., Golomb G.,
J. Control. Rel., 65, 221-229 (2000)

76) Sanchez A., Tobio M., Gonzalez L., Fabra A., Alonso M. J., Eur. J. Pharm. Sci., 18, 221-229 (2003).

77) Li Y. P., Pei Y. Y., Zhang X. Y., Gu Z. H., Zhou Z. H., Yuan W. F. Zhou J. J., Gao X. J., J. Control. Rel., 71, 203-211 (2001).

78) Allemann E., Leroux J. C., Gurny R., Adv. Drug Del. Rev., 34, 171189 (1998).

79) Benita M. B., Laloup M., Versteryhe S., Deurit J., Brackrleer J. D., Jongert E., Borchart G., Int. J. Pharm, 266, 17-27 (2003).

80) Aktas Y., Andrieux K., Alonso M. J., Calvo P., Gursoy R. N., Couvreur P., Capan Y., Int. J. Pharm., 298, 378-383 (2005).

81) Kim T. H., Park I. K., Nan J. W., Choi Y. J., Cho C. S., Biomaterials, 25, 3783-3792 (2004)

82) Uhrich K. E., Cannizzaro S. M., Larger R. S., Shakesheff K. M., Chem. Rev., 99, 3183-3198 (1999).

83) Ranney D. F., Biochem. Pharmacol., 59, 105-114 (2000).

84) Tobia M., Gref R., Sanchez A., Langer R., Alonso M. J., Pharm. Res. 15, 270-275 (1998).

85) Smith A., Hunneyball I. M., Int. J. Pharm., 30, 215-220 (1986)

86) Akiyoshi K., Kobayashi S., Shichibe S., Mix D., Baudys M., Kim S. W., Sunamoto J., J. Control. Rel., 4, 313-320 (1998).

87) Wang N., Wu X. S., Pharm. Dev. Technol., 2, 135-142 (1997).

88) Weng H., Zhou J., Tangm L., Hu Z., Biomater. Sci. Polym., 15 , $1167-1180$ (2004)

89) Kun N., Kwang H. L., You H. B., J. Control. Rel., 97, 513-525 (2004).

90) Pattou J. N., Palmer A. F., Biomacromolecules, 6, 2204-2212 (2005).

91) Vinognadov S., Batrakova E., Kabanov A., Colloids and Surfaces B. Biointerfaces, 16, 291-304 (1999).

92) Gupta M., Gupta A. K., J. Control. Rel., 99, 157-166 (2004).

93) Daeman T., Mare A. D., Bungener L., Jonge J. D., Huckriede A. Wilschut J., Adv. Drug Del. Rev., 57, 451-463 (2005).

94) Hillery A. M., Toth I., Shaw A. J., Florence A. T., Alexander T., J. Control. Rel., 41, 271-281 (1996).

95) Ramanathan S., Qiu B., Pooyan S., Zhang G., Stein S., Leibowitz M J., Sinko P. J., J. Control. Rel., 77, 199-212 (2001).

96) Hillery A. M., Pharm. Sci. Technol. Today, 1, 69-75 (1998).

97) Nishiyama N., Kataoka K., Adv. Exp. Med. Biol., 519, 155-177 (2003).

98) Savic R., Luo L., Eisenberg A., Maysinger D., Science, 300, 615618 (2003).

99) Torchilin V. P., J. Control. Rel., 73, 137-172 (2001).

100) Rosler A., Vandermeulen G. W. M., Klok H. A., Adv. Drug. Deliv Rev., 53, 95-108 (2001).

101) Jones M. C., Leroux J. C., Eur. J. Pharm. Biopharm., 48, 101-111 (1999).

102) Torchilin V. P., Lukyanov A. N., Gao Zhonggao., Sternberg P. B., Proc. Natl. Acad. Sci. U.S.A., 100, 6039-6044 (2003).

103) Mizamura Y., Matsumura Y., Hamaguchi T., Nishiyama N., Kataoka K., Kawaguchi T., Hrushesky W. J., Moriyasu F., Kakizoe T., Jpn. J. Cancer Res., 92, 328-336 (2001).

104) Nishiyama N., Bae Y., Miyata K., Fukushima S., Kataoka K., Drug Discov. Today: Technologies, 2, 21-26 (2005).

105) Tomalia D. A., Baker H., Dewald J., Hall M., Kallos G., Martin S., Roeck J., Ryder J., Smith P., Polym. J., 17, 117-132 (1985).

106) Aulenta F., Hayes W., Rannard S., Eur. Polym. J., 39, 1741-1771 (2003).

107) Padilla D. J., Omayra L., Ihre H. R., Gagne L., Frechet J. M. J., Szoka J., Francis C., Bioconj. Chem., 13, 453-461 (2002).

108) Orive G., Hernandez R. M., Gascon A. R., Pedraz J. L., Cancer Therapy, 3, 131-138 (2005).

109) Tripathi P. K., Khopade A. J., Nagaich S., Shrivastava S., Jain S., Jain N. K., Pharmazie, 57, 261-264 (2002).

110) Choi Y., Thomas T., Kotlyar A., Islam M. T., Baker J. R., Chem. Biol., 12, 35-43 (2005).

111) Kinhara F., Arima H., Tsutsumi T., Hirayama F., Uekama K., Bioconjug. Chem., 13, 1211-1219 (2002).

112) Sayed M. El., Ginski M., Rhodes C., Ghandehari H., J. Control. Rel., 81, 355-365 (2002).

113) Hermanson G. T., "Bioconjugate Techniques," Academic Press, San Diego, California, 1996.

114) Dwaine F. E., Christopher G. T., Expert Opin. Biol. Ther, 3, 655663 (2003). 
115) Sumner J. P., Aylott J. W., Monsona E., Kopelman R. A., Analyst (London), 127, 11-16 (2002).

116) Mirkin C. A., MRS Bull., 25, 43-54 (2000).

117) Schneider B. H., Dickinson E. L., Vach M. D., Hoizer J. V., Howard L. V., Biosensor Bioelectronics, 15, 13-22 (2000).

118) Darkow R., Groth T., Ajlbrecht W. M., Lutzow K. M., Paul D., Biomaterials, 20, 1277-1283 (1999).
119) Averitt R. D., Sarkar D., Halas N. J., Phys. Rev. Lett., 78, 4217-4220 (1997).

120) Sershen S. R., Westcott S. L., Halas N. J., West J. L., J. Biomed. Mater. Res., 51, 293-298 (2000)

121) Bashir R., Adv. Drug Del. Rev., 56, 1565-1586 (2004).

122) Niemeyer C. M., Review in Molecular Biotechnology, 82, 47-66 (2001). 